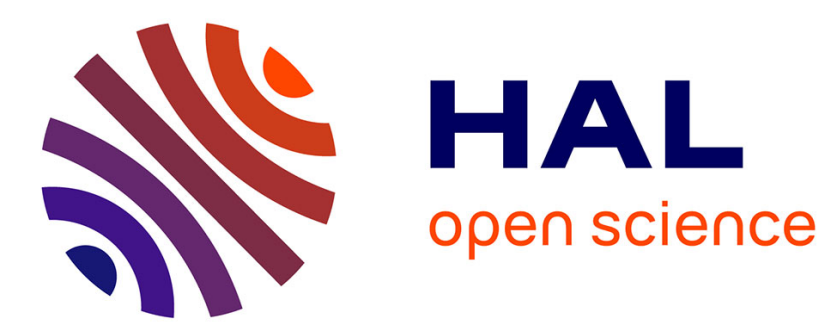

\title{
Démocratiser les musées : une profession intellectuelle au féminin
}

Aurélie Peyrin

\section{To cite this version:}

Aurélie Peyrin. Démocratiser les musées : une profession intellectuelle au féminin. Travail, genre et sociétés, 2008, 19, p. 65-85. 10.3917/tgs.019.0065 . halshs-01102536

\section{HAL Id: halshs-01102536 \\ https://shs.hal.science/halshs-01102536}

Submitted on 13 Jan 2015

HAL is a multi-disciplinary open access archive for the deposit and dissemination of scientific research documents, whether they are published or not. The documents may come from teaching and research institutions in France or abroad, or from public or private research centers.
L'archive ouverte pluridisciplinaire $\mathbf{H A L}$, est destinée au dépôt et à la diffusion de documents scientifiques de niveau recherche, publiés ou non, émanant des établissements d'enseignement et de recherche français ou étrangers, des laboratoires publics ou privés. 


\title{
DEMOCRATISER LES MUSEES. UNE PROFESSION INTELLECTUELLE AU FEMININ
}

\begin{abstract}
Aurélie Peyrin
$\mathrm{V}$

isites guidées, visites conférences, visites animées ou ateliers: les diverses formes d'accompagnement des visiteurs dans les musées se définissent par l'énonciation d'un discours savant sur les œuvres pour « instruire » le grand public. En France comme aux États-Unis, c'est à travers cette profession que les femmes sont entrées dans les muséesi au début du XXe siècle. Le portrait de groupe des chargé-e-s d'accompagnement a bien sûr évolué depuis, reflet des transformations de la condition féminine. Les diplômées d'histoire de l'art aujourd'hui ne sont plus seulement des jeunes bourgeoises cultivant les arts d'agrément et la culture littéraire et scientifique pour bien tenir leur rôle dans le mariage ; ces femmes, jeunes et moins jeunes, d'origine supérieure ou moyenne, souhaitent généralement fonder un foyer mais veulent aussi travailler pour s'épanouir, dans un métier stimulant. La profession a cependant conservé ses traits caractéristiques initiaux comme si, dès l'origine, l'activité d'accompagnement des visiteurs avait été pensée, conçue pour des femmes, fussent-elles «savantes ». De fait, le travail d'accompagnement n'a jamais été considéré comme donnant droit à un emploi à part entière, ni reconnu comme un «vrai » métier. Il s'agit en cela d'un cas singulier: cette profession intellectuelle exercée par des femmes très diplômées est aussi une profession dont les formes d'emploi sont précaires, dont l'activité est intermittente et que les protagonistes cherchent à faire reconnaître professionnellement.

L'accompagnement des visiteurs dans les musées forme ainsi l'activité centrale d'une profession féminine atypique, car d'emblée prise en charge par des femmes
\end{abstract}


savantes, qui conjugue cependant les traits typiques des métiers de femme et des emplois féminins. En termes de représentations sociales d'abord, il s'agit bien d'un "métier de femme » tel que l'a défini Michèle Perrot: l'activité de travail prolonge les qualités «naturelles » de la femme (bourgeoise), éducatrice et hôtesse hors pair, disponible et patienteii ; en termes de pratiques sociales ensuite, la précarité et le temps partiel sont deux particularités attachées à l'emploi féminin (Maruani, 2000 ; Angeloff, 2000). Étudier cette profession intellectuelle au féminin permet donc de réconcilier deux champs d'investigation habituellement disjoints : d'un côté, la problématique de la précarité et de la flexibilité du travail ou celle de la nonreconnaissance des qualifications sont associées aux emplois peu ou pas qualifiés (Maruani et Nicole, 1989) ; de l'autre, les problématiques d'inégalités d'accès et de carrière se concentrent sur les professions supérieures autour de la question de la féminisation et de sa prémisse, l'accès des femmes aux études supérieures (Cacouault-Bitaud, 2001).

L'article suit une trame chronologique ; les deux premières parties s'appuient sur des données d'archives, la troisième partie sur des données quantitatives et qualitatives ( $c f$. encadré Données et méthodes). Au début du Xxe siècle, accompagner les visiteurs dans les musées est une profession d'emblée conçue pour les femmes, et plus précisément conçue par les conservateurs hommes afin d'écarter des fonctions scientifiques les premières diplômées de l'Ecole du Louvre. $\mathrm{Au}$ fur et à mesure du développement de la fonction dans les musées publics français, ces femmes savantes ne sont pas restées silencieuses face aux conditions de travail et d'emploi qui leur étaient faites: elles se sont rassemblées en association, puis en syndicat dans les années 1950. Au début du XXI ${ }^{e}$ siècle, les caractéristiques de cette profession intellectuelle demeurent cependant très similaires à la situation en vigueur au début du XXe siècle. Ce qui a changé, c'est que ces conditions d'emploi flexibles attirent aussi certains hommes, essentiellement des artistes, qui s'en accommodent car ils y trouvent le temps libre nécessaire à leur travail de création. Comme les femmes qui exercent ce métier, ces hommes sont généralement dans une configuration familiale traditionnelle connue sous le nom de «breadwinner » : la plupart des chargé-e-s d'accompagnement ont un-e conjoint-e qui assure la sécurité des revenus du couple ou de la famille. La reconnaissance sociale de la profession est également toujours un enjeu majeur aujourd'hui. Quel que soit leur sexe, les chargé-e-s d'accompagnement tendent à naturaliser les compétences mises en œuvre dans l'emploi, sans doute en raison d'une prépondérance du diplôme dans la conception qu'ont les acteurs (recruteurs et employés) de la valeur professionnelle d'un-e chargé-e d'accompagnement.

\section{Données et méthodes}

Les chargé-e-s d'accompagnement sont étudié-e-s dans les musées publics, ayant toujours été employé-e-s par les collectivités publiquesiii : État, puis collectivités territoriales. Les archives des musées nationaux sont d'abord mobilisées pour retracer l'histoire de la profession de chargé-e d'accompagnement; les différentes cotes indiquées au fil du texte illustrent la variété et l'éclatement des sources de documentation à ce sujet (dossiers « École du Louvre », " Musée du Louvre ", « Visites conférences », " Service éducatif », etc.)

Le matériau quantitatif exploité ensuite est issu d'une enquête réalisée par la Direction des musées de France du ministère de la Culture et de la Communication et adressée aux quelques 1400 musées sous sa tutelle. Le questionnaire portait sur les personnels et moyens affectés à l'accompagnement. Parmi les 725 musées répondants, $493(68 \%)$ déclaraient au moins une personne affectée à cette fonction ; les 1931 individus ainsi désignés constituent la population 
Démocratiser les musées. Une profession intellectuelle au féminin

mère des données statistiques qui seront présentées dans la troisième partie. Ces données permettent d'établir un portrait statistique des chargé-e-s d'accompagnement et des services dans lesquels elles et ils exercent (les services des publics, services culturels et autres services éducatifs) en 2001, mais on s'intéresse ici particulièrement à la structure des formes d'emploi et du temps de travail.

Des entretiens biographiques complètent le tableau en restituant le vécu des actrices et acteurs sur leur travail et leur carrière. Réalisés entre 2002 et 2004, auprès d'environ soixante-dix chargé-e-s d'accompagnement d'une vingtaine de musées publics aux collections variées, et situés dans trois régions françaises, ces entretiens étaient centrés sur deux aspects : le parcours de l'individu jusqu'à l'emploi occupé d'une part, et les conditions de travail et d'emploi au moment de l'enquête d'autre part.

${ }^{3}$ L'activité d'accompagnement des visiteurs dans les musées est prise en charge par un autre groupe professionnel qu'on n'étudiera pas ici : les guides, guides-conférenciers et guides interprètes détenteurs d'une carte professionnelle délivrée conjointement par le ministère du Tourisme et le ministère de la Culture, qui exercent de fait dans les musées publics, où ils emmènent des groupes de visiteurs, mais en dehors des musées nationaux car leur activité est organisée par des associations, des offices municipaux et des sociétés privées de tourisme. Les deux groupes professionnels se distinguent donc d'abord par leurs employeurs, pour des raisons étonnantes : les collectivités publiques qui gèrent leurs propres musées ne recrutent pas les candidats détenteurs d'un titre certifiant leur compétence pour exercer ce métier, alors que la majorité de ceux-ci sont de fait autant diplômés, et dans des spécialités similaires, que les chargé-e-s d'accompagnement et chargé-e-s d'accompagnements employés par les collectivités publiques en tant qu'employés de musées. Par ailleurs, les guides sont eux aussi en majorité des femmes, et sont employés de façon précaire, à la fois temporaire et intermittente.

\section{UNE PROFESSION «SAVANTE » D'EMBLEE FEMINISEE : \\ L'ACCOMPAGNEMENT ENTRE DEUX GUERRES}

L'histoire de l'accompagnement des visiteurs est indissociable de celle de l'École du Louvre: le baccalauréat n'étant pas nécessaire dans cet établissement d'enseignement supérieur, des femmes s'y sont inscrites dès la fin du XIXe siècle. La question de la place des «femmes savantes» dans les musées a semble-t-il rapidement posé problème aux conservateurs des musées nationaux qui étaient alors tous des hommes et dont le corps était en cours de constitution dans la fonction publique d'État. L'accompagnement des visiteurs, mis en place entre les années 1920 et 1930, a rapidement été considéré comme une activité d'appoint pour étudiants et jeunes diplômés, avant d'être, pour ainsi dire, réservé aux femmes.

\section{L'École du Louvre, une opportunité pour les étudiantes de la fin du XIXe siècle}

L'École du Louvre fut créée en 1882 avec une double mission : former les futurs conservateurs et chargés de missions scientifiques de l'État d'une part, et offrir au grand public des leçons pratiques d'histoire de l'art d'autre part. L'ambition d'ouverture, similaire à celle de l'École Pratique des Hautes Études créée une quinzaine d'années auparavant, impliquait pour ses fondateurs l'accès sans conditions aux enseignements pour les auditeurs libres (le grand public), comme pour les élèves (destinés aux métiers scientifiques). Cette école, accessible sans le baccalauréat, représentait une aubaine pour certaines femmes de l'époque, étudiantes dans les lycées féminins dont le programme excluait les matières décisives pour l'accès aux études supérieures (Christen-Lécuyer, 2000). De fait, les pionnières $^{\text {iv }}$ étaient sans doute issues de la bourgeoisie, ce qui leur avait permis de 
financer des cours particuliers pour maîtriser les matières qui figuraient parmi les prérequis indispensables pour être élève de l'École du Louvrev : ces derniers devaient en effet avoir suivi «l'enseignement classique » et « connaître les langues anciennes et, parmi les modernes, l'allemand $»$ ii .

En une vingtaine d'années, les femmes ont quitté le rôle d'amatrices, auxquelles on proposait des connaissances conçues comme des arts d'agrément, pour celui de savantes, engagées dans une carrière professionnelle : la première thèse de l'École du Louvre accordée à une femme en 1901 atteste que « la période non fonctionnelle [des études] est révolue»(Christen-Lécuyer, 2000). Au tournant du siècle, les femmes sont donc entrées dans la compétition (entre elles, mais surtout contre les hommes) pour les postes de conservateurs et autres chargés de mission sur les chantiers de fouilles à l'étranger. La concurrence pour ces emplois était assez rude à l'époque: la fonction publique était en cours d'élaboration et les emplois de conservateurs (fonctionnaires) étaient rares, même pour les hommes ; de nombreux diplômés de l'École du Louvre débutaient leur carrière comme "commis", premier grade de l'échelle administrative, ou patientaient pendant plusieurs années sans revenus, avec le seul titre $\mathrm{d}^{\prime}$ " attaché libre des musées nationaux »vii.

\section{L'accompagnement,}

\section{une activité d'appoint parfaite pour une femme?}

La mise en place d'une offre d'accompagnement des visiteurs dans les musées nationaux, à partir des années 1920, résulte de la conjonction de trois éléments distincts. Les visites guidées en langues étrangères et en français proposées par des guides indépendants dans, puis aux abords du Louvre (peut-être depuis l'ouverture des collections au public, les archives font défaut avant le XVIIII siècle), ont représenté un véritable repoussoir pour les conservateurs des musées nationaux. Les "conférences-promenades" organisées dans les grands musées anglais et américains étaient présentées, quant à elles, comme le modèle à imiter. Les visites dirigées de l'École du Louvre, cours magistraux dans les salles des musées, devant les œuvres mêmes, formaient enfin la trame des visites proposées au grand public.

Dès leur mise en place, des visites guidées furent proposées aux anciens étudiants et diplômés de l'École du Louvre. En créant cette offre de services aux visiteurs, les conservateurs et professeurs de l'École du Louvre souhaitaient disposer d'une source de revenus pour leurs anciens élèves, en attendant que soient créés des postes de conservateurs ou d'attachés (assistants rémunérés). Cependant, alors même que les élèves masculins étaient nettement majoritaires (les effectifs des promotions étaient encore très faibles dans les années 1920-1930), les archives montrent que les visites furent aussitôt prises en charge par des femmes, anciennes élèves ou diplômées de l'École. Précisément, les premières «dames guides » furent recrutées sur examen en 1931.

Le directeur des musées nationaux déclarait n'avoir, dans les années 1930, «aucune objection pour les femmes. Leur emploi a donné dans les musées d'excellents résultats. »viii Comme les demoiselles des PTT au tournant du siècle, les femmes furent d'autant plus facilement acceptées qu'elles étaient "cantonnées dans une classe qui ne porte pas ombrage" aux hommes (Bachrach, 1987). La constitution de la première association professionnelle des conservateurs de musées en 1923, soit juste après la création des visites guidées dans les musées, 
Démocratiser les musées. Une profession intellectuelle au féminin

peut d'ailleurs être interprétée comme une réaction à l'émergence de la concurrence féminine dans les musées. La relégation des femmes dans des fonctions de médiatrices a rendu la mixité possible dans les musées car elle réglait le problème de la concurrence féminine pour l'accès aux fonctions scientifiques sur un marché du travail restreint, en orientant les femmes vers une voie spécifique.

La procédure de recrutement des chargé-e-s d'accompagnement était très sélective, traduisant l'exigence intellectuelle des conservateurs vis-à-vis de cette fonction d'accompagnement : les candidat-e-s, ancien-ne-s élèves et diplômé-e-s de l'École du Louvre, devaient prouver l'étendue de leurs connaissances sur les collections des musées nationaux, devant un jury de conservateurs, professeurs à l'École. À l'issue de cette sélection, le titre de "conférencier des musées nationaux » leur permettait de conduire des visites officielles et de percevoir une rémunération à la tâche (à la visite) : les « conférencières » se partageaient les recettes des visites entre elles, après ponction de la part réservée à la Réunion des musées nationaux pour les frais $\mathrm{d}^{\prime}$ organisation ${ }^{\mathrm{ix}}$.

Cette organisation du travail et de l'emploi initiale est, encore aujourd'hui, la caractéristique paradoxale de cette profession intellectuelle. Le système d'emploi à la demande, assorti d'un salaire irrégulier et imprévisible, s'il semble correspondre aux impératifs d'un métier de femme dans les représentations sociales (peu accaparant), ne répond apparemment pas aux souhaits des conférencières ellesmêmes, comme elles l'expriment dès les années 1950.

\section{LA LENTE MISE EN PLACE D'UN CADRE D'EMPLOI : DES ANNEES 1950 AUX ANNEES 1990}

Après les années 1930, les archives des musées nationaux sont lacunaires: de nombreux documents ont sans doute disparu pendant la seconde guerre mondiale et les musées ont été fermés à plusieurs reprises pendant le conflit, avant une réouverture partielle sous l'occupation. Lorsqu'on retrouve les chargé-e-s d'accompagnement dans les archives des années 1950, le contexte du droit du travail a changé: l'insécurité matérielle induite par ces conditions d'emploi atypiques contraste désormais avec l'emploi assorti d'une protection sociale « universelle».

\section{Des femmes syndiquées pour faire reconnaître leur droit à la protection sociale}

L'hypothèse d'une moindre tendance à la revendication dans les professions féminisées (Cacouault-Bitaud, 2001) n'est pas validée ici. Après la Seconde guerre mondiale, les «conférencières des musées nationaux » revendiquèrent la reconnaissance de leur profession ${ }^{x}$. Sur le plan symbolique, elles voulaient la liberté de choisir leur discours (ne pas réciter des textes écrits par les conservateurs), mais manifestèrent aussi un certain corporatisme : elles voulaient restreindre l'accès à la profession aux seul-e-s diplômé-e-s de l'École du Louvre. Sur le plan pratique, elles réclamaient en outre une revalorisation salariale (elles voulaient bien travailler plus, mais uniquement si elles étaient payées plus) et une amélioration de leurs conditions de travail (diversifier les publics, disposer d'une salle de repos au sein du Louvre ${ }^{\mathrm{x} i}$ ). Ces revendications demeurant lettre morte, les 
conférencières créèrent une association en 1962, qu'elles transformèrent en syndicat sous l'égide de la Confédération générale des cadres l'année suivante. Elles conservaient les mêmes revendications, mais menaçaient désormais leur employeur de se mettre en grève.

Cette première manifestation collective, dont les archives ont conservé la trace, a opportunément coïncidé avec le mouvement d'extension d'un régime de protection sociale à l'ensemble des salariés, initié après la seconde guerre mondialexii. Du début des années 1950 à la fin des années 1960, la correspondance conservée dans les archives des musées nationaux rend compte de la forte pression exercée par la nouvelle administration de la Sécurité sociale sur celle des musées nationaux. Cette dernière a en effet longtemps regimbé contre l'assujettissement des salaires des conférencières à ces prélèvements sociaux, multipliant les arguments pour démontrer que le travail d'accompagnement n'était pas un emploi à part entière.

Le premier argument mis en avant fut l'intermittence du travail. En 1954, l'administration proposa seulement trois noms à l'immatriculation sur vingt-deux conférenciers, puisque les autres "n'atteign[ai]ent pas une durée de travail de 60 heures par trimestre ». En 1964, l'administration précisa à nouveau que le travail des conférencières était «temporaire », d'abord parce qu'elles ne travaillaient pas toute l'année - les vacances scolaires étaient chômées dès cette époque - et ensuite parce que la Réunion des musées nationaux ne pouvait pas leur assurer un travail régulier, et donc un salaire fixe - la multi-activité était alors déjà quasiment imposée. Les représentations des gens de musées sont résumées sous la plume d'un directeur de la Réunion des musées nationaux: les conférenciers sont "rétribués comme vacataires, ce qui rend leur position identique à celle de tel artisan qui loue ses services ici et là »xiii. Le deuxième type d'argument repose sur la forte majorité féminine de cette population, qui joue, comme on s'y attend, en défaveur de l'immatriculation : quand elles ne sont pas déjà assujetties à la sécurité sociale par leur employeur principal, les conférencières en «bénéficient par leur conjoint». Puisqu'il ne s'agit que d'une activité d'appoint, il serait donc inutile, pour l'employeur, de leur accorder quelque droit social.

L'administration de la Sécurité sociale ayant systématiquement affirmé que chacun des arguments était irrecevable à ses yeux, de nouvelles formules contractuelles furent progressivement imaginées par l'administration des musées nationaux, en conservant l'objectif d'une exonération de l'affiliation au régime général. Dans les années 1960, les contrats de travail, généralement signés pour un an, précisaient notamment les engagements de part et d'autre en termes de volume horaire : l'administration des musées nationaux s'engageait à recourir en priorité aux conférenciers sélectionnés jusqu'à concurrence d'un certain volume horaire via une procédure dite de "concours " (l'ancien examen), et ceux-ci s'engageaient en échange à se rendre disponibles pour assurer ce volume horaire.

Petit à petit, l'accompagnement est ainsi rentré dans le cadre de la législation du travail, sous l'action conjuguée des conférencières des musées nationaux - qui ne sont pas restées passives face à cette situation et ont revendiqué de meilleures conditions de travail et d'emploi - et de la Sécurité sociale, qui a forcé l'administration des musées nationaux à assumer son rôle d'employeur vis-à-vis des conférencières. 
Démocratiser les musées. Une profession intellectuelle au féminin

\section{La transformation du monde des musées}

Après les années 1970, les musées ont connu un véritable âge d'or : rénovation d'anciens bâtiments, enrichissement des collections, création de nouveaux établissements et élargissement des champs thématiques, etc. Tous ces bouleversements ont été accompagnés d'une modernisation des modes de gestion (Ballé et Poulot, 2004) et d'une féminisation rapide et massive des professions de conservateur (Octobre, 2001) et de gardien. Au-delà, toute la philosophie des musées a progressivement évolué vers une reconnaissance des visiteurs, par une meilleure prise en compte des conditions d'accueil et de mise en exposition des objets (Eidelman, 2005 ; Poulard, 2005). Ces transformations ont eu deux types de répercussions sur l'activité d'accompagnement: l'effectif dédié à l'accompagnement a d'une part été multiplié par quatre entre 1975 et 2001, passant de 475 à 1918 personnes dans les musées territoriaux xiv ; la Loi relative aux musées de France, votée en 2002, a d'autre part inscrit les missions d'accompagnement au cœur des missions permanentes des musées soutenus par l'État, apportant une reconnaissance politique à cette activité professionnelle.

Les conditions de travail et d'emploi des chargé-e-s d'accompagnement ont néanmoins traversé cette période de mutations en conservant de fortes similitudes avec celles observées au début du XXe siècle. À la fin des années 1980, la multiplication de l'offre d'accompagnement dans les musées publics s'est opérée au prix d'arrangements statutaires peu satisfaisants, puisqu'aucun corps ni cadre d'emploi n'étaient encore susceptibles d'accueillir les chargé-e-s d'accompagnement. Les femmes constituant la profession se sont à nouveau rassemblées pour agir : l'association Médiateurs du patrimoine fut créée en 1987 par Marie-Thérèse Caille (conférencière du Louvre recrutée et formée par Germaine Cart), autour d'un noyau de femmes exerçant des fonctions d'accompagnement dans les musées et monuments publics. En s'appuyant sur une enquête adressée à « environ 300 musées ayant une activité culturelle », ses représentantes revendiquaient (à leur tour) la professionnalisation de l'accompagnement. En dépit d'un diplôme au moins égal à la licence dans les deux tiers des cas, les statuts occupés étaient infiniment variés et nécessitaient donc une «réhabilitation administrative » des situations « les plus invraisemblables (vacataires, travailleurs intellectuels temporaires - sic -, gardiens de square...) ou tributaires de situations administratives précaires ou provisoires, comme seul notre génie sait en créer (conférenciers des monuments nationaux, de la Caisse nationale des monuments historiques et des sites, enseignants mis à disposition ou en heures de décharge de service) »xv.

\section{Un inventaire à la Prévert : \\ le recensement des « statuts » des médiateurs en 1987}

«Un travailleur d'utilité collective ; quarante-trois vacataires ; dix-huit contractuels ; quarante et un titulaires (six conservateurs, douze adjoints au conservateur, cinq rédacteurs, cinq commis, quatre attachés, deux Education nationale, deux sans activité, un ouvrier professionnel, un assistant technique, une secrétaire administrative, un auxiliaire, un grade spécifique). Soit $41 \%$ de titulaires, dont presque $20 \%$ ont un statut municipal "fourre-tout». [...] Les quelque $60 \%$ de personnels vacataires ou contractuels méritent quelques précisions : les hypothèses avancées sont que ces personnels travaillent depuis de nombreuses années dans ces structures et veulent y faire carrière, mais qu'ils n'ont comme solution que de préparer des concours administratifs qui ne correspondent pas aux postes qu'ils aspirent à occuper. » Extrait du comte rendu de l'enquête présentée lors des Troisièmes rencontres internationales pour la protection du patrimoine culturel, 18, 19 et 20 novembre 1987, Avignon. 


\section{La reconnaissance de « nouvelles » fonctions muséales dans les années 1990}

Les revendications des chargé-e-s d'accompagnement des musées nationaux et territoriaux des années 1950 aux années 1980 ont finalement été entendues dans les années 1990. C'est en effet à cette époque que trois chargées de mission, au sein du service central (issu du service des visites guidées de 1928) de la Direction des musées nationaux du ministère de la Culture, se sont attachées à « professionnaliser » l'accompagnement. Leur projet, ambitieux, portait sur trois aspects complémentaires: mettre en place des cadres d'emploi dans la fonction publique pour accueillir des "médiateurs culturels", alimenter ces emplois par des diplômés spécialisés en "médiation culturelle », et légitimer ces diplômes par un dispositif théorique et conceptuel (Caillet et Lehalle, 1995 ; Caillet et Coppey, 2004).

Des négociations s'engagèrent alors entre le ministère de la Culture et le Centre national de la fonction publique territoriale pour aménager trois des nouveaux cadres d'emploi créés dans la filière culturelle territoriale en $1991^{\text {xvi }}$ : aux épreuves orales $\mathrm{du}$ concours d'attaché de conservation du patrimoine (catégorie $\mathrm{A}$ ), d'assistant de conservation du patrimoine et d'assistant qualifié de conservation du patrimoine (catégorie B), les candidats peuvent désormais choisir la médiation parmi quatre options (conservation, médiation, histoire des institutions de France, sources documentaires). L'évolution du cadre réglementaire a donc rendu statutairement possible le recrutement de chargé-e-s d'accompagnement fonctionnaires dans des cadres d'emplois adaptés. Ceci étant, ces cadres d'emploi ne semblent pas être identifiés par les employeurs : quinze ans après leur création, ils sont encore peu utilisés par les collectivités territoriales pour recruter les chargée-s d'accompagnement. Par ailleurs, les formations existent désormais dans de très nombreuses universités mais leurs diplômé-e-s ne sont pas embauché-e-s pour occuper les postes d'accompagnement. La faible diffusion du vocabulaire de la médiation parmi les chargé-e-s d'accompagnement, qui préfèrent se dire « animatrice » ou " conférencière », est enfin un indice d'une faible appropriation de la rhétorique institutionnelle sur la profession, même parmi les jeunes générations.

\section{LA PERSISTANCE DE PRATIQUES ET REPRESENTATIONS HERITEES DU DEBUT DU SIECLE}

Vacations, contrats à durée déterminée d'un à trois ans, un emploi jeune (cinq ans), ou même emploi de fonctionnaire : l'accompagnement se conjugue presque toujours au temps incomplet. Le temps partiel et le travail à la demande répondent en effet à une logique productive à la fois ancienne et durable : l'organisation du travail $\mathrm{d}$ 'accompagnement est tout entière tournée vers la réponse à la demande des «clients ", essentiellement des groupes scolaires (Peyrin, 2007). L'activité des musées suit traditionnellement la saisonnalité scolaire, avec des périodes «mortes » (toutes les vacances scolaires, surtout les deux mois d'été) car l'activité des services des publics s'adresse très rarement aux touristes, pris en charge par des chargé-e-s d'accompagnement extérieurs aux musées, détenteurs de cartes professionnelles de guide ou conférencier ${ }^{\mathrm{xvii}}$. 
Démocratiser les musées. Une profession intellectuelle au féminin

\section{La prépondérance des formes d'emploi précaires et du travail intermittent}

Depuis la création de la fonction dans les musées, la forme d'emploi privilégiée des chargé-e-s d'accompagnement a toujours été une curieuse hybridation publique entre indépendance et salariat : la vacation. Ce mode d'emploi spécifique à la fonction publique est une survivance de l'emploi à la tâche. Le terme vacataire désigne des "agents recrutés au moyen de contrats à durée déterminée d'une durée inférieure à un an pour l'exécution d'un acte déterminé ou d'une tâche ponctuelle. »xviii Les vacataires sont traités comme des salariés par le droit du travail (leurs employeurs et eux-mêmes sont assujettis aux cotisations de sécurité sociale), mais leur degré de protection est proportionnel à leur activité : une chargée d'accompagnement vacataire rencontrée au cours de l'enquête a par exemple découvert, au cours de sa grossesse, qu'elle ne bénéficierait pas de l'indemnisation de son congé légal de maternité faute d'avoir assez travaillé, donc assez cotisé. En outre, les vacataires et leurs employeurs ne cotisent pas au système d'assurance chômage. Leur protection est donc très inférieure à celle des salariés de droit commun et des agents publics.

Comme au début du siècle, les chargé-e-s d'accompagnement sont aujourd'hui en majorité des salarié-e-s instables : en 2001, $39 \%$ étaient vacataires, $21 \%$ en emploi aidé et $9 \%$ en contrat à durée déterminée, contre seulement $28 \%$ en contrat à durée indéterminée ou fonctionnaire ( $c f$. tableau 1). Les hommes, qui représentent un quart de l'effectif des chargé-e-s d'accompagnement recensés par l'enquête de la DMF, sont un peu moins souvent en emploi instable que les femmes.

Tableau 1 : Distribution sexuée des chargé-e-s d'accompagnement selon la forme d'emploi

\begin{tabular}{lcccc}
\hline & selon la forme d'emploi & $\begin{array}{c}\text { Taux de } \\
\text { féminisation } \\
\text { des différentes } \\
\text { formes } \\
\text { d'emploi }\end{array}$ & $\begin{array}{c}\text { Effectif } \\
\text { total }\end{array}$ \\
\hline Vacataire & $39 \%$ & Hommes & $35 \%$ & 238 \\
\hline Emploi aidé & $21 \%$ & $20 \%$ & $75 \%$ & 130 \\
\hline CDD & $9 \%$ & $8 \%$ & $78 \%$ & 55 \\
\hline Total emploi instable & $69 \%$ & $63 \%$ & $76 \%$ & 423 \\
\hline CDI & $3 \%$ & $4 \%$ & $73 \%$ & 22 \\
\hline Fonctionnaire & $24 \%$ & $28 \%$ & $72 \%$ & 158 \\
\hline Enseignant & $1 \%$ & $1 \%$ & $71 \%$ & 5 \\
\hline Total emploi stable & $28 \%$ & $33 \%$ & $67 \%$ & 21 \\
\hline Autre & $3 \%$ & $4 \%$ & $\mathbf{7 5} \%$ & $\mathbf{6 2 9}$ \\
\hline Total & $\mathbf{1 0 0} \%$ & $\mathbf{1 0 0} \%$ & & 5 \\
\hline
\end{tabular}

Source : enquête DMF, 2001.

Champ : chargé-e-s d'accompagnement, non-réponses (23\%) et sexe non renseigné (12\%) exclus. Lecture : les pourcentages en colonne permettent de comparer la distribution des hommes et des femmes par forme d'emploi. Si les différences sont faibles, les chargées d'accompagnement sont tout de même moins souvent en emploi stable que leurs homologues masculins (28\% contre $33 \%$ ). Le taux de 
féminisation par forme d'emploi permet de visualiser la concentration des femmes et des hommes sur certaines formes d'emploi plutôt que d'autres, opposant ainsi le CDD, très majoritairement féminin, au statut d'enseignant mis à disposition qui l'est nettement moins.

Par ailleurs, la distribution des postes par sexe (cf. tableau 2) indique elle aussi des différences, faibles mais significatives: moins souvent affectés aux postes d'accueil et d'accompagnement, les hommes sont en revanche deux fois plus souvent professeur-relais que les femmes. Par ailleurs, ils ne sont pas plus souvent chefs de service que les femmes, qui confirment leur supériorité numérique à tous les échelons de la hiérarchie des services des publics ${ }^{\mathrm{xix}}$. 
Démocratiser les musées. Une profession intellectuelle au féminin

Tableau 2 : Distribution sexuée des chargé-e-s d'accompagnement selon la fonction

\begin{tabular}{lcccc}
\hline & \multicolumn{2}{c}{$\begin{array}{c}\text { Distribution } \\
\text { selon la fonction }\end{array}$} & $\begin{array}{c}\text { Taux de féminisation } \\
\text { des différentes fonctions }\end{array}$ & Effectif total \\
\cline { 2 - 5 } & Hommes & Femmes & & 172 \\
\hline Accueil & $9 \%$ & $13 \%$ & $77 \%$ & 822 \\
\hline Accompagnement & $51 \%$ & $58 \%$ & $74 \%$ & 219 \\
\hline Professeur-relais & $25 \%$ & $11 \%$ & $53 \%$ & 148 \\
\hline Chef de service & $9 \%$ & $10 \%$ & $73 \%$ & 110 \\
\hline Support technique & $6 \%$ & $8 \%$ & $78 \%$ & $\mathbf{1 4 7}$ \\
\hline total & $\mathbf{1 0 0} \%$ & $\mathbf{1 0 0} \%$ & $\mathbf{7 1 \%}$ & \\
\hline
\end{tabular}

Source : enquête DMF, 2001.

Champ : ensemble des personnes recensées dans le service des publics, autre réponses et non réponses exclues $(n=259)$.

Seulement un tiers des chargé-e-s d'accompagnement travaille à temps plein, la moitié répondant au coup par coup aux sollicitations de son employeur, ce qui reflète et amplifie le résultat du tableau $\mathrm{n}^{\circ} 1$ (certains contrats à durée déterminée ou indéterminée offrent en effet une souplesse horaire assimilant en pratique l'activité de leurs détenteurs à la vacation). La distribution sexuée selon le temps de travail ( $c f$. tableau 3) montre à nouveau de faibles différences entre les sexes, les hommes étant un peu plus souvent à temps plein que les femmes.

Tableau 3 : Distribution des chargé-e-s d'accompagnement selon le temps de travail

\begin{tabular}{lcc}
\hline & Femmes & Hommes \\
\hline sur demande & $52 \%$ & $51 \%$ \\
\hline temps partiel & $16 \%$ & $15 \%$ \\
\hline temps plein & $29 \%$ & $33 \%$ \\
\hline sans précision & $3 \%$ & $1 \%$ \\
\hline Total & $\mathbf{1 0 0} \%$ & $\mathbf{1 0 0} \%$ \\
\hline
\end{tabular}

Source : enquête DMF, 2001.

Champ : chargé-e-s d'accompagnement, $\mathrm{N}=922$.

Les recherches sur le temps partiel, notamment celles de Margaret Maruani, ont nettement démontré les conséquences salariales du travail à temps incomplet. Le salaire des vacataires étant strictement déterminé par le nombre d'heures travaillées, il varie considérablement d'un mois à l'autre. Les chargé-e-s d'accompagnement vacataires ont une rémunération horaire élevée : en 2002, le salaire horaire brut était par exemple de $24 €$ dans un grand musée municipal de la région Rhône-Alpes ou encore de $37 €$ dans un grand musée parisien ${ }^{x x}$. Les interviewé-e-s estimaient leurs revenus à $800 €$ par mois en moyenne, sans compter les mois «creux », c'est-à-dire sans travail et sans revenus, en juillet et août, et les mois «faibles» qui comprennent une période de vacances scolaires (essentiellement février, avril et décembre). En pratique, si le montant horaire 
semble prendre en compte le niveau d'études et la qualification du travail, le revenu obtenu par ce biais n'est qu'un revenu d'appoint, qui ne permet pas aux vacataires de vivre de cette seule activité et les contraint de chercher d'autres employeurs, voire d'autres activités. Les configurations familiales sont également un élément important compte tenu de ces faibles salaires : lorsqu'ils ou elles sont en couple, les chargé-e-s d'accompagnement peuvent généralement compter sur le salaire d'un monsieur ou d'une madame "gagne-pain » en emploi stable (cadre de la fonction publique ou du secteur privé pour les conjoints, enseignantes pour les conjointes).

Le temps partiel observé ici n'a cependant pas tout à fait les mêmes caractéristiques que sur le marché du travail en général. Les chargé-e-s d'accompagnement sont tou-te-s très diplômé-e-s; tandis que les statistiques de l'emploi établissent clairement que le temps partiel tend à diminuer avec le niveau de diplôme (Maruani et Meulders, 2005). Le temps partiel et le travail à la demande correspondent ici à une tradition organisationnelle faiblement remise en cause. De plus, le temps partiel des chargé-e-s d'accompagnement contractualisés est souvent négocié. Dans le cadre d'un processus de déprécarisation initié par les collectivités territoriales ${ }^{x \times i}$, l'employeur affecte par exemple un nombre d'heures équivalent temps plein à l'équipe du service des publics, à charge pour chaque membre d'exprimer la quotité horaire souhaitée. Dans les deux cas observés, en Alsace et en Rhône-Alpes, l'offre de l'employeur impliquait nécessairement le choix d'un temps de travail incomplet par la majorité des membres de l'équipe. Dans ces même deux cas, la plupart des chargé-e-s- d'accompagnement ont accepté un temps partiel (à $70 \%$ ou à $80 \%$ le plus souvent) ; les seul-e-s à avoir demandé un temps complet faisant état de leur nécessité de disposer d'un «vrai » salaire pour être autonomes (le seul homme et les quelques femmes concerné-e-s étaient célibataires). Ce consentement s'explique par ailleurs par le maintien de stratégies de multi-activité élaborées par les vacataires (Peyrin, 2007): les chargé-e-s d'accompagnement, auxquel-le-s la municipalité a proposé ces emplois à temps incomplet, ont été (plus ou moins longtemps) vacataires et cumulaient souvent plusieurs emplois, dans d'autres musées ou dans l'enseignement supérieur; l'accroissement $d u$ volume horaire $d^{\prime} u n$ de leurs emplois aurait donc impliqué l'abandon d'autres activités, non seulement rémunératrices mais également considérées comme nécessaires à leur épanouissement professionnel.

Cette profession intellectuelle exercée par des femmes diplômées est donc paradoxalement touchée par les stigmates de l'emploi féminin peu qualifié: précarité et temps partiel (Maruani, 2000), dont les diplômes sont justement censés protéger leurs détenteurs et détentrices. Cette situation perdure depuis près d'un siècle, alors même que les cadres statutaires ont évolué dans la fonction publique. L'accompagnement des visiteurs dans les musées est un exemple de la persistance de représentations sociales attachées aux activités féminines, même les plus qualifiées. L'analyse de la composante métier de cette profession va nous permettre de compléter le tableau: malgré la multiplication de formations universitaires spécialisées en médiation culturelle dans les années 1990, les critères de recrutement ont peu évolué depuis le début du siècle : la survalorisation des diplômes se fait au détriment de la reconnaissance des compétences professionnelles acquises en cours d'emploi. 
Démocratiser les musées. Une profession intellectuelle au féminin

\section{Une survalorisation des diplômes constante...}

Des années 1930 aux années 1960, les chargé-e-s d'accompagnement des musées nationaux ont été recruté-e-s sur deux types de critères : l'exactitude et l'étendue des connaissances, ainsi que la qualité de l'expression orale, critères évalués au cours d'un examen devant un jury composé de conservateurs. Si elles reposent toujours sur ces deux critères initiaux, les procédures de recrutement se sont complexifiées au fil du temps et avec la diffusion de l'activité dans les musées territoriaux : elles se sont fragmentées en plusieurs étapes et enrichies de nouveaux acteurs. L'analyse des discours et témoignages recueillis dans une vingtaine de musées fait apparaître des "conventions de compétences » (Eymard-Duvernay et Marchal, 1997) relativement homogènes d'un endroit à l'autre, et quelle que soit la spécialité du musée.

La première étape du processus de recrutement est la sélection sur dossier (curriculum vitae et lettre de motivation), présélection généralement opérée par le ou la responsable du service des publics. Le CV doit attester d'une certification par l'État d'un niveau d'études supérieures supérieur ou égal à bac +3 , mais aussi d'une spécialisation (une période historique par exemple), repérable à travers le sujet de mémoire (en Master) ou de thèse (en Doctorat). La pratique des langues vivantes est appréciée, mais est très rarement considérée comme obligatoire en raison des visiteurs ciblés par les services des publics. De façon sous-jacente, les recruteurs privilégient certains profils à cette étape de la sélection, surtout pour le recrutement de vacataires : les étudiant-e-s et jeunes diplômé-e-s, les artistes, ou les mères de jeunes enfants, c'est-à-dire des individus qui ne cherchent qu'un emploi d'attente ou un emploi d'appoint. Ce critère de sélection rarement explicité permet notamment aux employeurs de minimiser le risque de revendications syndicales.

La seconde étape du recrutement consiste en un entretien devant un jury tripartite, qui rassemble représentants de la conservation (chef d'établissement), du service des publics (chef de service), et de l'administration territoriale (employeur). Selon François Eymard-Duvernay et Emmanuelle Marchal, la présence de différents acteurs permet de maintenir une tension entre une pluralité de façons de juger. Mais tout se passe en réalité comme si les représentants de la conservation et du service des publics se disputaient entre eux la légitimité de juger les candidats, tout en s'alliant pour imposer leurs propres critères à l'employeur. Lorsque les conservateurs dominent le jury, l'entretien est une réplique de l'oral du concours de conservateur du patrimoine, durant lequel les connaissances du candidat sont mises à l'épreuve. Selon Laurence, 37 ans, chargée d'accompagnement d'un grand musée d'art dans la région Rhône-Alpes, le jury était "un vrai tribunal d'universitaires » qui s'est livré à un " canardage de questions sans fin ». Lorsque le service des publics domine le jury, en revanche, ses représentant-e-s mettent l'accent sur une évaluation de la personnalité du candidat: Pamela, adjointe de la responsable du service des publics d'un grand musée d'art alsacien, dit par exemple chercher à évaluer "si les gens sont passionnés ou pas. [...] On ressent dans le face à face si la personne est chaleureuse, souriante ».

Soixante-dix ans après la mise en place de l'accompagnement dans les musées, les conservateurs gardent une position prééminente dans les jurys de recrutement : les chargé-e-s d'accompagnement ont toujours été recruté-e-s sur les mêmes profils que les conservateurs et valorisent leurs diplômes de la même manière. Les chargée-s d'accompagnement tentent d'affirmer leur position dans les musées par le 
diplôme et par la légitimité scientifique. Mais, malgré une revendication professionnelle précoce, ils et elles ne parviennent pas à combler le fossé entre services des publics et services de conservation qui prévaut encore. Les conservateurs écrivent les textes scientifiques selon les critères canoniques de l'histoire de l'art (les notices, les catalogues) et les chargé-e-s d'accompagnement écrivent les textes de vulgarisation (petit journal, cartels allongés). Il est possible qu'en s'attachant à la reconnaissance scientifique, alors même que la parité était progressivement conquise dans la conservation, les chargé-e-s d'accompagnement aient minimisé l'importance de compétences spécifiques à l'activité d'accompagnement, se privant par là même d'une forme de légitimité essentielle pour la profession (Peyrin, 2006).

\section{... au détriment de « compétences naturalisées »}

D'un entretien à l'autre, les chargé-e-s d'accompagnement racontent leur «apprentissage sur le tas », à travers l'échange avec leurs collègues, avec les enseignants, et face au public. Au moment de l'enquête, aucun-e n'avait suivi de formation validant institutionnellement les compétences professionnelles. La plupart des interviewé-e-s estiment d'ailleurs qu'une formation préalable ne rendrait pas compte de la réalité de l'échange avec le public.

« Rien ne remplace le contact avec le public.», Elsa, 28 ans, médiatrice multimédia en contrat emploi jeune, musée d'art, Ile-de-France.

«Les enfants m'ont tout appris » Jeanne-Marie 60 ans, conservatrice bénévole d'un petit musée de site, Ile-de-France (conférencière du Louvre à partir des années 1960).

Étant donné le mode de recrutement, les chargé-e-s d'accompagnement nouvellement embauché-e-s appréhendent en effet assez vaguement la tâche qui leur incombe, qu'ils ou elles découvrent en observant les chargé-e-s d'accompagnement plus expérimenté-e-s. Bien souvent, les nouvelles recrues ne savent guère comment se préparer et font appel à leurs compétences estudiantines : elles lisent des ouvrages sur les œuvres et les artistes, apprennent par cœur des éléments historiques, biographiques, stylistiques, autant d'éléments constitutifs de l'analyse d'œuvre en histoire de l'art. Mais une fois face au public, elles se rendent compte que leur métier repose essentiellement sur la parole : il faut gérer ses émotions pour maîtriser sa voix et positionner son corps dans l'espace, entre les œuvres et les visiteurs. Pour la plupart des chargé-e-s d'accompagnement, cette compétence d'orateur relève $\mathrm{du}$ don, $\mathrm{du}$ talent, c'est-à-dire d'une qualité individuelle « naturelle ».

«J'ai naturellement le contact facile », Winona, 42 ans, chargée d'accompagnement vacataire, musée d'art, Rhône-Alpes.

«J'aime séduire », Nils, 35 ans, chargé d'accompagnement contractuel à 70 \%, musée d'art, Alsace.

Accompagner les visiteurs ne consiste, en outre, pas seulement à «étaler sa science " sur les œuvres (Sonia, 27 ans, chargée d'accompagnement vacataire, musée d'art, Rhône-Alpes), mais à faire partager plusieurs approches possibles d'une œuvre d'art à un public toujours différent, rarement diplômé en histoire de l'art. Il faut donc faire usage de techniques pédagogiques, structurer le discours de façon logique en apportant progressivement des éléments, s'appuyer sur les connaissances préalables des visiteurs pour développer un aspect inattendu... Encore une fois, la compétence pédagogique est renvoyée aux bonnes volontés 
Démocratiser les musées. Une profession intellectuelle au féminin

individuelles: seul-e-s certain-e-s interviewé-e-s manifestent le souci de se documenter sur la question des publics.

« Je crois que c'est une question de connaissances, mais pas seulement, c'est aussi la façon de les porter aux autres, une sensibilité, une écoute du public. [...] C'est une question de caractère, de personnalité. » Bérengère, 52 ans, responsable du service des publics d'un musée d'art, contractuelle à mi-temps, Ile-de-France.

De nombreux-ses interviewé-e-s disent mettre en œuvre dans leur travail des qualités «humaines », liées à la "personnalité »; cela correspond précisément, dans les entretiens, à des termes comme l'«ouverture», la «sensibilité », $l^{\prime}$ «attention» aux autres et la «volonté de communiquer», le «besoin de transmettre $»$.

«Comme j'ai formé pas mal de gens, c'est justement la grosse question : est-ce que ça s'apprend ?

$\mathrm{C}^{\prime}$ est un grand débat qu'on a. J'ai quand même la conviction qu'il y a quelque chose qui est de

l'ordre de l'intuitif et quelque chose qu'on a en soi. [...] Il y a quand même quelque chose, une espèce de vocation, on va dire. Après, ça s'affine, il y a des choses qu'il ne faut pas faire, des choses qu'on apprend à ne pas faire ou à faire. » Isabelle, adjointe à la responsable du service des publics, attachée de conservation titulaire à plein-temps, musée d'art, Alsace

La capacité d'écoute et d'attention au public, caractéristique très attachée au genre féminin dans les représentations, est (selon les interviewé-e-s) la principale qualité professionnelle des chargé-e-s d'accompagnement. La rhétorique basée sur la passion, la vocation de la relation et de la transmission, énoncés par les interviewé-e-s comme des critères d'épanouissement professionnel (Erlich, 2001), traduit aussi de la même façon un rabattement de la compétence professionnelle sur des qualités intrinsèquement liées à l'individu et - implicitement - à son sexe. Ce type de discours fragilise la construction du rôle de médiateur au sein du musée car il nie la dimension professionnelle des compétences développées lors de l'exercice du travail, qui vont pourtant croissant avec l'expérience

L'accompagnement met en jeu des capacités relationnelles, de communication et de diplomatie difficiles à évaluer (tant par les salariés que par les employeurs) parce qu'elles s'acquièrent plutôt par l'expérience et la socialisation que par des formes structurées ${ }^{x i i}$. On retrouve également dans cette invisibilité du travail de mise en scène de soi-même, cette négation du travail de la voix et du corps, les processus à l'œuvre pour les chanteuses de jazz (Buscatto, 2003). Contrairement au monde du jazz où des "conceptions sexuées de la musique séparent instrumentistes et chanteuses " (p.35) le discours des hommes ne se distingue pas vraiment de celui des femmes: comme elles, ils naturalisent les compétences engagées dans le métier. Précisons néanmoins que certaines femmes et certains hommes n'adhèrent pas tout à fait au discours naturalisant et cherchent à développer leurs compétences en acquérant des techniques de communication et de pédagogie par la formation et la documentation. Ceux-là se lassent d'ailleurs moins vite du métier que les autres, qui s'en remettent au «charme» ou au «talent .

L'accompagnement est une profession féminine «savante » depuis le début du $X X^{e}$ siècle ; une profession savante mais considérée comme un travail d'appoint, d'une part, et inféodée aux conservateurs (qui étaient alors en majorité des 
hommes), d'autre part. Si l'accompagnement des visiteurs dans les musées fut la première profession muséale à accueillir les pionnières de l'École du Louvre dans les années 1900 il fut aussi une voie de relégation féminine, loin des prétentions scientifiques qu'elles exprimaient; une relégation dans un travail présentant des caractéristiques typiques des métiers et emplois dits féminins: compétences naturalisées et donc non reconnues, emplois instables et intermittents, profession socialement peu valorisée.

Tout au long du Xxe siècle, les femmes qui ont exercé cette fonction d'accompagnement des visiteurs dans les musées ont tenté de vivre de ce travail, malgré les piètres conditions d'emploi qui le caractérisent, et d'en tirer le meilleur parti pour construire leur parcours professionnel. En enquêtant sur l'histoire des employé-e-s du Grand Bazar, Anne-Sophie Beau (2002) a montré que l'instabilité de la main-d'œuvre n'était pas «une invention du XXe siècle». L'exemple des conférencier-e-s des musées nationaux enrichit cette histoire de l'emploi public instable ; il montre par ailleurs que la mise en place d'un cadre réglementaire ne suffit pas à venir à bout de pratiques de travail et d'emploi fondées sur des représentations sociales.

L'exercice de la profession d'accompagnement oscille, depuis l'origine, entre deux extrêmes: le travail transitoire - une étape dans une carrière qui amène à des fonctions scientifiques - et le travail d'appoint - en plus et à côté d'une autre activité conçue comme symboliquement prépondérante, comme la pratique artistique par exemple - (Peyrin, 2007). Toutefois, être chargé-e d'accompagnement n'a plus aujourd'hui tout à fait le même sens qu'au début du siècle, quand la profession de conservateur était fermée aux femmes : les femmes se dirigeaient alors vers ce travail faute d'avoir accès aux professions scientifiques; c'est désormais plutôt le déséquilibre entre le nombre de candidats et le nombre de postes ouverts aux concours qui les oriente vers ces fonctions. La profession de conservateur a en effet connu une féminisation lente mais irrépressible sur la même période : environ les deux tiers des conservateurs du patrimoine (territoriaux) sont des femmesxxiii. Entre une profession d'emblée féminine (l'accompagnement), une profession féminisée (la conservation) et une profession mixte (le gardiennage), les musées constituent désormais un système des professions (Abbott, 1988) féminin, ou mieux: un monde féminin, car les musées sont par ailleurs fréquentés par une majorité de femmes (Donnat, 2001).

\section{BIBLIOGRAPHIE}

Аввотт Andrew, 1988, Le système des professions, Chicago, University of Chicago Press.

ANGELOFF Tania, 2000, Le temps partiel : un marché de dupes?, Paris, Syros.

ANXo Dominique, Flood Lennart et Kocoglu Yussuf, 2002, "Offre de travail et répartition des activités domestiques et parentales au sein du couple : une comparaison entre la France et la Suède », Économie et statistique, n 352-353, pp. 127-150.

BACHRACH Susan, 1987, « La féminisation des PTT au tournant du siècle », Le Mouvement social, $\mathrm{n}^{\circ} 140$, pp. 69-87.

Balle Catherine et POUlot Dominique, 2004, Musées en Europe. Une mutation inachevée, Paris, La documentation Française.

BEAU Anne-Sophie, 2002 «Les salarié-e-s du grand commerce: des "employé-e-s" ? Les parcours professionnels des salarié-e-s du Grand Bazar de Lyon aux XIXe et XXe siècles ", Travail, genre et sociétés, $\mathrm{n}^{\circ} 8$, pp. 55-72.

16 A Travail, genre et sociétés n 19 - Avril 2008 
Buscatto Marie, 2003, «Chanteuse de jazz n'est point métier d'homme. L'accord imparfait entre voix et instrument », Revue française de sociologie, vol. 44, n 1 , pp. 35-62.

Cacouault-Bitaud Marlaine, 2001, « La féminisation d'une profession est-elle le signe d'une baisse de prestige?", Travail, genre sociétés, $\quad \mathrm{n}^{\circ} 5$, pp. 93-115.

CAILlET Elisabeth et LEHALLE Evelyne, 1995, A l'approche du musée : la médiation culturelle, Lyon, Presses universitaires de Lyon.

CAILlET Elisabeth et COPPEY Odile, 2004, Stratégies pour l'action culturelle, Paris, L'Harmattan.

CHRISTEN-LECUYER Carole, 2000, « Les premières étudiantes de l'Université de Paris », Travail, genre et sociétés, $\mathrm{n}^{\circ} 4$, pp. 35-50.

DAUNE-RICHARD Anne-Marie, 2001, «Hommes et femmes devant le travail et l'emploi », in Blöss, T. (dir.) La dialectique des rapports hommes-femmes, Paris, PUF, pp. 127-150.

DONNAT Olivier, 2001, "La féminisation des pratiques culturelles », in Blöss, T. (dir.) La dialectique des rapports hommes-femmes, Paris, PUF, pp. 423-431.

EIDELMAN Jacqueline, 2005, Musées et public : la double métamorphose, Habilitation à diriger les recherches, Université Paris 5 - René Descartes.

ERLICH Valérie, 2001, «Entrée dans l'enseignement supérieur et manières d'étudier », in Blöss, T. (dir.) La dialectique des rapports hommes-femmes, Paris, PUF, pp. 89-101.

EYMARD-DUVERNAY François et MARCHAL Emmanuelle, 1997, Façons de recruter. Le jugement des compétences sur le marché du travail, Paris, Métailié.

GADREY Nicole, 1995, "Formation, qualification et mixité », in Ephesia, La place des femmes. Les enjeux de l'identité et de l'égalité au regard des sciences sociales, Paris, La Découverte, pp. 552-556.

MARUANi Margaret, 2006 [2000], Travail et emploi des femmes, Paris, La Découverte.

MARUANI Margaret et MEULDERS Danièle, 2005, "Chômage, sous-emploi et précarité ", in Maruani, Margaret (dir.) Femmes, genre et sociétés. L'état des savoirs, Paris, la Découverte, pp. 227-236.

MaruANi Margaret et NiCOLE Chantal, 1989, Au labeur des dames. Métiers masculins, emplois féminins, Paris, Syros.

OCTOBRE Sylvie, 2001, "Construction et conflits de la légitimité professionnelle: qualification et compétences des conservateurs de musées », Sociologie du travail, vol. 43, n 1, pp. 91-109.

PERROT Michèle, 1987, «Qu'est-ce qu’un métier de femme ? », Le mouvement social, n 140, pp. 3-8.

PEYRIN Aurélie 2006, «Flexibilité de l'emploi et reconnaissance sociale des savoirs et savoir-faire. Une analyse des conditions de possibilité d'une identité professionnelle.", Savoir, travail et société / Knowledge, Work and Society, Vol. 4, n³, pp. 105-129.

PEYRIN Aurélie 2007, "Les usages sociaux de l'emploi précaire. Le cas des médiateurs de musées », Sociétés contemporaines, $\mathrm{n}^{\circ} 67, \mathrm{pp} .7-26$.

POULARD Frédéric, 2005, Les musées de collectivités, les conservateurs et la mise en place des politiques culturelles, thèse de sociologie, Université Paris 8-Vincennes Saint-Denis.

SAINT MARTIN (DE) Monique, 1989, «Structure du capital, différenciation selon les sexes et 'vocation' intellectuelle », Sociologie et sociétés, vol. XXI, n² 2, p. 9-25.

SCHWEITZER Sylvie, 2002, Les femmes ont toujours travaillé, Paris, Editions Odile Jacob.

WEISS Louise, 1968, Une petite fille du siècle, Paris, Albin Michel.

ZOLBERG Vera, 1989, "Le musée des beaux-arts, entre la culture et le public : barrière ou facteur de nivellement? », Sociologie et sociétés, vol. XXI-2, pp. 75-90.

\footnotetext{
i Depuis la fin du XIX ${ }^{\circ}$ siècle, les femmes d'Amérique du nord se sont massivement engagées dans les musées comme fondatrices ou chercheuses, souvent bénévoles, mais ont rapidement été cantonnées aux postes d'éducation, et exclues des programmes scientifiques (Zolberg, 1983 et 1989, cf. aussi les communications de Brian Young et Terry Rudell, historiens canadiens, lors d'un séminaire francocanadien du 7 février 2003 sur l'évolution des fonctions du monde muséal, au Muséum d'histoire naturelle).

ii «Les 'métiers de femmes', ceux dont on dit qu'ils sont 'bien pour une femme' » sont le fruit d'une « construction et [le] produit du rapport des sexes ». Plusieurs ingrédients les composent : ils sont
} 
d'abord « réputés peu accaparants », ils « s'inscrivent dans le prolongement des fonctions 'naturelles', maternelles et ménagères », ils reposent enfin sur « des qualifications réelles déguisées en 'qualités 'naturelles et subsumées dans un attribut suprême : la féminité. » (Perrot, 1987, pp. 4-5)

iii L'activité d'accompagnement des visiteurs dans les musées est prise en charge par un autre groupe professionnel qu'on n'étudiera pas ici : les guides, guides-conférenciers et guides interprètes détenteurs d'une carte professionnelle délivrée conjointement par le ministère du Tourisme et le ministère de la Culture, qui exercent de fait dans les musées publics, où ils emmènent des groupes de visiteurs, mais en dehors des musées nationaux car leur activité est organisée par des associations, des offices municipaux et des sociétés privées de tourisme. Les deux groupes professionnels se distinguent donc d'abord par leurs employeurs, pour des raisons étonnantes : les collectivités publiques qui gèrent leurs propres musées ne recrutent pas les candidats détenteurs d'un titre certifiant leur compétence pour exercer ce métier, alors que la majorité de ceux-ci sont de fait autant diplômés, et dans des spécialités similaires, que les chargé-e-s d'accompagnement et chargé-e-s d'accompagnements employés par les collectivités publiques en tant qu'employés de musées. Par ailleurs, les guides sont eux aussi en majorité des femmes, et sont employés de façon précaire, à la fois temporaire et intermittente.

iv Les premières élèves furent deux sœurs, Aline et Elisabeth Wattel en 1886. Mlle Bengesco fut ensuite la première femme à réussir les examens en 1891, Mlle Paschoud la première femme à présenter la thèse de l'Ecole en 1901. Source : archives des musées nationaux, Ecole du Louvre : cotes *1FF Résultat des examens, 2 registres, 1884-1911 et *2FF Inscriptions (cartes de cours des élèves et auditeurs), 14 registres, 1886-1930.

v Le premier volume de l'autobiographie de Louise Weiss (1968) rend bien compte de l'état des possibles pour ces jeunes femmes éduquées, généralement promises à la profession d'enseignante, agrégées dans le meilleur des cas, venant rejoindre « le prolétariat des institutrices des établissements privés » dans le pire des cas.

vi Source : archives des musées nationaux, projet pour la constitution d'une école d'administration des musées nationaux, 1882, cote F1 Organisation et historique de l'Ecole du Louvre.

vii Le titre $\mathrm{d}^{\prime}$ « attaché libres des musées nationaux » était décerné aux élèves diplômés de l’Ecole du Louvre ayant présenté une thèse avec succès. Ceux-ci s'engageaient à se tenir à disposition des musées nationaux pendant dix ans dans l'éventualité d'un emploi, et exerçaient souvent comme bénévoles. viii Lettre du Directeur des musées nationaux datée du 23 février 1931. Cf. aussi l'idée développée par Paul Leroy-Baulieu à la fin du XIX ${ }^{\circ}$ siècle, selon laquelle « les femmes ont d'instinct la connaissance de l'enfance ", cité par Michèle Perrot, 1987 (Leroy-Baulieu Paul, 1888, Le travail des femmes au XIX` siècle, Paris, Charpentier).

ix La Réunion des musées nationaux (créée en 1895 pour acheter les œuvres et enrichir les collections nationales), et la Direction des musées nationaux (dont le directeur est alors aussi le Directeur de l'Ecole du Louvre), sont deux entités distinctes réunies sous la tutelle du ministère de l'Instruction publique et des Beaux-Arts.

× A travers leur responsable, Germaine Cart, qui devint par la suite conservatrice des musées nationaux et dont l'action après guerre et jusqu'à la fin des années 1960 contribua à organiser et développer l'accompagnement muséal en direction des enfants.

xi Elles ne l'obtiendront qu'en 1965.

xii Le principe d'une Sécurité sociale obligatoire pour les salariés (créée le 4 octobre 1945), a été généralisé à tous les français, salariés ou non, le 22 mai 1946.

xiii Archives des musées nationaux, cote 6 AA, 2 (c) Service éducatif ; Rétribution des conférenciers ; relèvement des tarifs ; budget des visites conférences ; affiliation des conférenciers à la sécurité sociale (1963-1966). Prévisions budgétaires pour 1963.

xiv L'effectif dédié à l'accompagnement dans les musées territoriaux en 1975 a été répertorié par MarieFrançoise Poiret la même année pour l'Inspection des musées nationaux ; les données concernant l'année 2001 sont issues de la base de données du département des publics du ministère de la Culture. xv Lettre adressée par Marie-Thérèse Caille, présidente de l'association au ministre de la Culture, le 28 novembre 1987.

xvi Décret n91-855 du 2 septembre 1991.

xvii Pour assurer un travail continu aux chargé-e-s d'accompagnement, d'autres stratégies seraient possibles, et notamment une stratégie d'offre consistant à développer des services à destination de " clients » suivant une saisonnalité inverse : les centres de loisirs par exemple, mais aussi les familles, ou encore les retraités, les comités d'entreprise, etc. Certains musées s'engagent progressivement dans cette voie, sous l'impulsion des collectivités territoriales. 
xviii Arrêt du Conseil d'Etat, mars 2003. Dans les faits, les syndicats de la fonction publique dénoncent régulièrement la présence de " vacataires permanents » dans l'Education nationale ou dans les établissements publics.

xix Ceci vient contrebalancer quelques prénotions des observateurs de la vie muséale : on entend souvent dire par des chercheurs et représentants institutionnels que les rares hommes réussiraient mieux leur carrière que les femmes dans cette profession. Cette idée repose uniquement sur quelques cas d'hommes ayant atteint des positions particulièrement visibles (responsable d'une association de médiateurs de musées ou d'un grand service des publics dans le plus grand musée d'une ville par exemple), autant d'arbres qui cachent la forêt des carrières féminines réussies.

xx Pour information, le SMIC horaire était d'un montant de 7,19€ brut la même année.

xxi Loi n96-1093 du 16 décembre 1996, dite « Loi Perben ».

xxii Anne-Marie Daune-Richard (2001), rappelant les résultats d'une enquête menée par Danièle Kergoat, dit à ce propos : «Ces considérations s'appliquent particulièrement aux compétences considérées comme féminines : acquises dans la sphère privée de la famille et dans l'exercice de rôles domestiques féminins d'attention aux autres, elles sont perçues par les employeurs mais aussi, souvent, par les salariées elles-mêmes comme des qualités "naturelles", attachées à leur identité personnelle et féminine et ne relevant pas - tout au moins pas immédiatement - du rapport salarial. »

xxiii Exactement $61 \%$ des conservateurs du patrimoine sont des femmes, ainsi que $72 \%$ des attachés de conservation du patrimoine, $88 \%$ des assistants qualifiés de conservation du patrimoine et des bibliothèques, $79 \%$ des assistants de conservation du patrimoine et des bibliothèques, cf. le rapport $\mathrm{La}$ fonction publique territoriale. Perspectives démographiques par région, CNFPT/CRACL, septembre 2001. Cette inversion du genre de la profession a souvent été signalée dans les publications qui y sont consacrées, sans jamais faire l'objet d'un traitement à part entière (Octobre, 2001 ; Poulard, 2005). 